\title{
A Dynamic Node Degree Management Scheme for Energy-efficient Routing Protocols in Wireless Ad Hoc Networks*
}

\author{
Yi-Cheng Huang, Sheng-Yan Chuang and Sheng-De Wang \\ Department of Electrical Engineering \\ National Taiwan University \\ Taipei, TAIWAN \\ sdwang@ntu.edu.tw
}

\begin{abstract}
In mobile devices, the battery-based power is a precious resource. To maximize the network lifetime becomes a challenge issue in MANETs. We found that transmission collision is a problem which affects the energy saving much. If we can reduce the numbers of the transmission collision, we save more power. In this paper, we propose a new mechanism to address this problem and hence prolong network lifetime. Our approach, based on Relative Neighborhood Graph (RNG), adjusts the transmission range according to the degree of the node which is only based on local information. We also suggest appropriate transmission power ranges to some routing protocols such as AODV, DSR and BELLMAN-FORD. Simulation results show that a great improvement of the energy saving for those routing protocolss when comparing with the performance of those protocols without applying our mechanism.
\end{abstract}

Keywords: ad hoc network, energy consumption, RNG, collision.

\section{Introduction}

A wireless ad hoc network is a collection of mobile wireless hosts or devices forming a network without any infrastructure or centralized access point. Mobile hosts communicate with each other by using a peer to peer manner. Besides that, ad hoc networks have other characteristics, such as quick deployment, self-organizing, self-managing, limited resource and so on. Ad hoc network mechanism is useful for many different fields because of its quick deployment property, those fields include personal area networks, home area networks, military purpose (battlefields), emergency rescues and so on.

In recent years, there have been many studies on ad hoc networks. Because network nodes are mobile, they cannot be line-powered. Instead, batteries are the main power source for them. Since battery power is limited, how to minimize energy consumption to prolong the network's lifetime is a big issue. There have been many researches on energy consumption in ad hoc networks [4-5] [14-15]. In [14], J. E.
Wieselthier et al. reduced the overall energy consumption in broadcasting and multicasting problems to increase the life time of ad hoc networks. One of their approaches is to perform energy-efficient multihop broadcasting. The other is the broadcast incremental power (BIP) algorithm which is used to build a minimum-energy broadcast tree. Both the approaches based on the global information. In [4], X. Chen, M. Faloutsos and S.V. Krishnamurth proposed a method to reduce the overall power consumption per broadcast. Every node attempts to adjust its transmission power level based on local (two-hop neighborhood) information. Their method can reduce the energy consumption and eliminate redundant transmissions as that of a scheme in which every node uses the default maximum power level for transmissions. C. Tang, C. S. Raghavendra and V. Prasanna proposed an energy-efficient adaptation, based on clustering scheme [5]. A node is assigned to be a head node in each cluster. The head node acts as a forwarding agent for its cluster members. All head nodes form a supernode backbone. Data multicast with three steps i) a sender node transmits data to its head node; ii) the data travels along the supernode backbone; iii) receivers finally obtain the multicast data from the cluster head node within their clusters.

For the sake of balancing power consumption, cluster nodes take turn to be a cluster headnode by using some strategies. J. Wu and F. Dai proposed a distributed solution to solve a high computation cost in a dense network [15]. Their solution is based on reducing the density of a mobile ad hoc network, consisting of two methods: clustering and adjustable transmission range. The key idea of adjustable transmission range is clusterheads forward the broadcast message with different ranges.

\subsection{Motivation}

In an ad hoc network the power is limited, so it is a kind of precious resource. A node spends much energy when it is in the active mode. Therefore, our main objective is to reduce the energy by minimizing the frequency of the collision when transmissions in each node. 
There are packet collisions if no collision avoiding mechanism is used (exposed station and hidden station). According to the collision problems we know that the number of the neighbors affects the frequency of the collision. A node with more neighbor nodes has the higher probability of the packet collision than the nodes with less neighbor nodes. Each collision costs at least two retransmissions (best case, one retransmission for each node and only two nodes collide). The cost of success delivery a packet increases. It means that each node consumes more energy when the collision rate is high. In this paper, we address this problem by shortening the transmission range to avoid collision when the number of the neighbors is large and hence reduce the frequency of collision.

\subsection{Problem Description}

There are no other studies discussing on the preventing from transmission collision in the ad hoc network to save the power consumption yet. We think the collision problem efforts much on power consumption. Our goal is that prolongs the network life time while maintains the connectivity of whole network.

In [8], they save power by reducing the energy consumption on communications interfaces and entering sleep mode when it is idle to extend battery lifetime for mobile hosts. In [16], B. Chen et al. measured power consumption of the Cabletron 802.11 network card. They summarized the power consumed by the card as follows:

- Tx : $1400 \mathrm{~mW}$

- Rx : $1000 \mathrm{~mW}$

- Idle : $830 \mathrm{~mW}$

- Sleeping : $130 \mathrm{~mW}$

These outcomes closely match the results obtained by Feeney and Nilsson [17]. Feeney and Nilsson also measured power consumption of a similar 802.11 network interface card in the ad hoc mode. We found that the energy at Tx and Rx modes is more than at Idle mode. According to the motivation mentioned in the previous section, we propose that a mechanism, based on Relative Neighborhood Graphs (RNG) [2-3], can save energy. We can get the information of adjacent nodes by one hop. The neighborhood information we collect includes node id, its neighbor node, and its degree. When a node obtains the neighborhood information from others, it can detect the signal strength of the received packet to determine the distance between source and the destination node. Besides it can be aware of the distance between every adjacency node and its neighbors. According to the information, we can determine the RNG topology. Our method can guarantee each node can have a path to any other node in the network and fewer collisions. In routing protocols [6] [12], they use broadcast to perform the route discovery process. Therefore, we use routing protocols to evaluate the performance of our algorithm. And the simulation results show that the routing protocols with our algorithm outperform those without our approach.

The rest of this paper is organized as follows: we discuss the design and properties of our algorithm in section 2. We show the simulation results and the conclusions in the section 3 and section 4, respectively.

\section{Algorithm}

In this section, we will describe our algorithm in detail. Our method not only decreases the node degree which can reduce collision, but also guarantee network connectivity. As we have discussed before, we found that RNG has been applied to many studies in ad hoc networks. In [2], he concluded RNG is a connected and planar graph. Consequently, RNG can guarantee network connectivity. We purpose an algorithm which is based on RNG and extend the local information from one-hop neighborhood information to two-hop neighborhood information. Our algorithm has three objectives: 1) to minimize node degree; 2) to reduce overall power consumption; and 3) as well as guarantee network connectivity.

\subsection{Communication Model and Assumption}

We model our network as a graph $\mathrm{G}=(\mathrm{V}, \mathrm{E})$, where $\mathrm{V}$ is the set of nodes and $\mathrm{E}$ is the edge set. We call that an edge $(u, v)$ belongs to $\mathrm{E}$ if and only if $u$ is communicable with $v$. The following list is our assumption:

1) Initially, network is connected and the transmission range is the same, i.e. there is no partition node. Every node can reach any other node in the network.

2) Each node has limited battery power.

3) Each edge is directed as shown in Figure 1.

4) We assume that each node can change its transmission power (tx_power) and the range can be $0<=$ tx_power $<=$ MAX_POWER, where MAX_POWER is the default maximum power level. Node $u$ can hear node $v$ if $u$ is within the transmission range of $v$.

5) We assume that no coordinator is in our network model, so interference is unavoidable.

\subsection{The Medium Channel Model}

We use the channel model the same as the power law model in [4], [11-12] and [16]: $\mathrm{P}_{\mathrm{rec}}=\mathrm{P}_{\mathrm{tx}} / \mathrm{r}^{\mathrm{n}}\left(\mathrm{P}_{\mathrm{rec}}\right.$ is the received power, $\mathrm{P}_{\mathrm{tx}}$ is the transmission power, $r$ is the transmission range and $n$ is a positive real value) In general, the value of $n$ is between 2 and 4, moreover, it depends on the characteristics of the communication medium and is often used to model the power 
attenuation with distance [4]. We assume nodes use omni-directional antennas. In [10] [13], there are more detailed models that reflect shadowing effects and antenna gains.

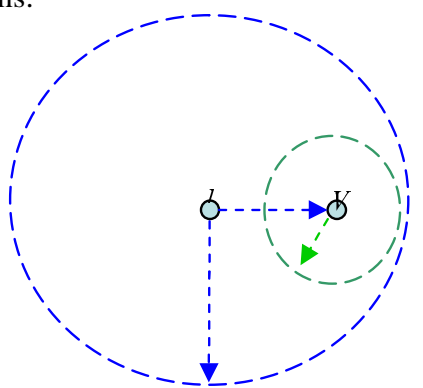

Figure 1. The directed edge $(\boldsymbol{u}, \boldsymbol{v})$ belongs to E, but edge $(\boldsymbol{v}, \boldsymbol{u})$ does not belong to $\mathrm{E}$

\subsection{The Design of Our Algorithm}

\subsubsection{Information exchanging phase}

At beginning all nodes send beacon signals by using default maximum power level. We assume that nodes exchange their neighbors' information by sending beacons while measure signal strengths of incoming messages and estimate distance between nodes and itself. And then they append its neighbor information to beacon. Each node collects the information of neighbors which are within two hops. That information includes the distances to all neighbors and the distances between one-hop neighbors and two-hop neighbors.

By the signal strength of the received packet, we can calculate the power level needed to reach a node. In fact, a node then attempts to choose its transmission power level to reach nodes within its relative neighborhood graph.

\subsubsection{Build RNG}

In this paper, we use the relative neighborhood graph (RNG) [2] as our localized approach. RNG is a geometric and graph-theoretic concept. The definition of RNG is as follows. Given a set of nodes in the plane, we say that an edge $(u, v)$ belongs to the RNG if there is no other node inside lune $(u, v)$, where lune $(u, v)$ is the intersection area of the interiors of the two circles centered at $u$ and $v$ with radius $\mathrm{d}(u, v)$. Figure 2 shows the example. We use the one of any RNG algorithm to build RNG. Each node uses the default maximum power level to send the beacon signal at first. Before it broadcasts the beacon message, it will adjust its transmission power. Our algorithm reduces the node degree by limiting each node's neighbors that belong to RNG (V). Each node does not start the establishing the RNG until it has gotten its neighborhood information.

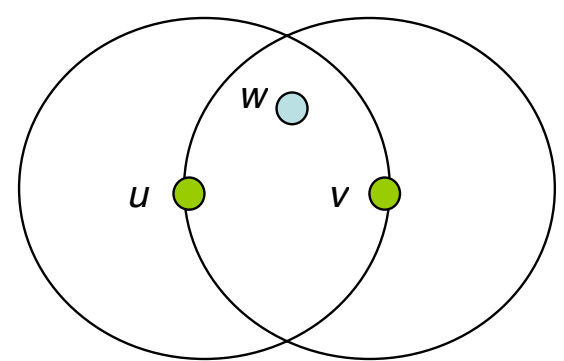

Figure 2. Edge $(u, v)$ is not in RNG because the node $w$

\subsubsection{Establishing an RNG neighborhood table}

After RNG of the network is established, each node set up its own RNG neighborhood table. The RNG neighborhood table provides RNG with neighbor information, including node id and received power (the signal strength), that is used to estimate the distance. The table lists in ascending order based on the received power of each node. Therefore we can make sure that the first node in the list is the farthest neighbor node. Accordingly, every node can choose its transmission power in order to cover its all RNG neighbors.

\subsubsection{Set up new transmission range}

Now each node can adjust its own transmission range according to RNG neighborhood table. The adjusting action is shown in Figure 3. At first, node $i$ use the default maximum transmission power to send messages. After node $i$ establishes its RNG neighboring nodes, for example $u$ and $v$, and then it chooses the node $u$ from its RNG neighborhood table. If the target degree is 2 , node $i$ adjusts its transmission range to include nodes $u$ and $v$.

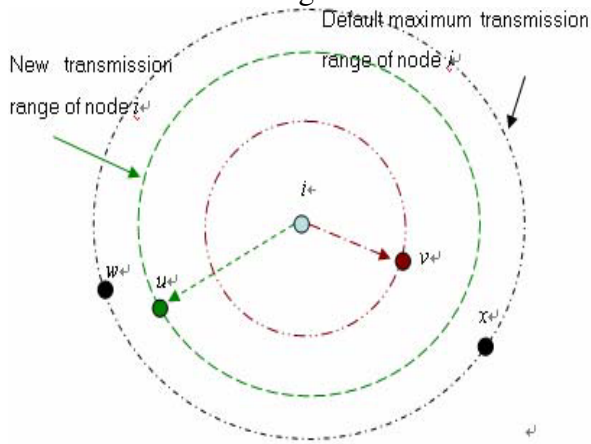

Figure 3. The illustration of adjusting transmission range

\subsubsection{Modify transmission range}

After we perform the set-up new transmission range procedure, it is found that RNG exists in asymmetric links. Figure 4 is an example of asymmetric link. Nodes $i$ and $x$ exist in asymmetric link as shown in 
Figure 4(a). Node i has two RNG neighbors as nodes $u$ and $v$; then it choose its transmission power so that its transmission range includes nodes $u, v$, and node $x$. Edge $(x, i)$ is not in RNG because of the node $v$. That is to say, node $x$ only has one RNG neighbor as node $v$. Consequently, edge $(i, x)$ is in RNG, but edge $(x, i)$ is not in RNG. There exists an asymmetric link between nodes $x$ and $i$. We have two choices to solve this situation which is shown as follow:

i) Each node discards messages from any node which is not its RNG neighbors and adjusts transmission range to include it. That is, when node $\mathrm{x}$ receives the message from node $\mathrm{i}$, it discards the message. It only delivers the message from node $\mathrm{v}$.

ii) The other method is whenever a node receives a broadcast message from its neighbors; it checks its transmission range to ensure that it includes this neighbor. That is to say, when node $\mathrm{x}$ receives the message from node $i$, it acquires the signal strength of the received packet. Then it checks its transmission range to ensure that it includes node $i$. The new transmission range of node $\mathrm{x}$ is shown in Figure 4(b).

\section{Simulation}

\subsection{Simulation Environment}

We use the GlomoSim [11] to evaluate the routing protocol performances of collision and energy consumption with and without our algorithm. The simulation parameters are listed in Table 1 . The network size is $N$ nodes, $\mathrm{N}=\{50,55,60, \ldots, 150\}$, those nodes are randomly and statically distributed over a 1400 unit $\times 1400$ unit area. We use the default medium type RADIO-ACCNOISE. We choose $15 \mathrm{dBm}$ as the default power. We compare routing protocols with three mechanisms. There are original RNG that only accepts message from RNG neighbors (RNG-1), extended RNG that guarantee symmetric links (RNG-2), and without our approach (non-RNG). For the non-RNG simulation of routing protocols, all nodes use the default power level during the simulation. AODV [12] is the chosen as the routing protocol. AODV is source-initiated on demand routing protocols. We randomly choose nodes that use FTP applications to transfer 20 files. The number of transferring file nodes is 10 pairs. All simulation results in this section are the averages of ten runs on different randomly-chosen nodes.

\subsection{Simulation Results}

\subsubsection{Collision}

Then we compare the collision of routing protocols with RNG-1, RNG-2 and Non-RNG, as shown in Figure 5. When the number of nodes increases, the collision increases. We randomly choose ten pairs of nodes to simulate the FTP protocol. The source node transmits 20 files to the destination node. We calculate $A$ (all collisions through the simulation) and $B$ (collisions through nodes performing the beacon phase). Then we perform $A-B$ to get the collisions for transmitting files. We observe that RNG-1 outperforms RNG-2, since the average node degree of RNG-2 is bigger than that of RNG-1, because RNG-2 can guarantee that the all nodes pairs have symmetric links.

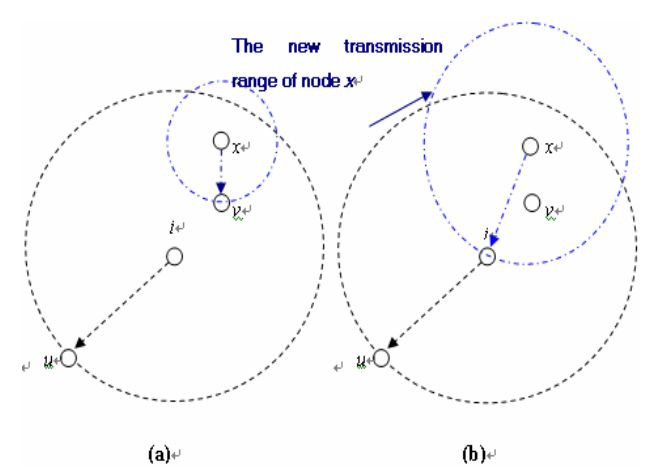

Figure 4. (a) The asymmetric link example (b) the new transmission range of node $x$

Table 1. Simulation parameters

\begin{tabular}{|l|l|}
\hline TERRAIN-DIMENSIONS & 1400 x 1400 square units \\
\hline Number-Of-Nodes & $50 \sim 150$ \\
\hline Radio type & RADIO-ACCNOISE \\
\hline Node replacement & Random \\
\hline Max. radio tx power & $15 \mathrm{dBm}$ \\
\hline RADIO-RX-SENSITIVITY & $-91.0 \mathrm{dBm}$ \\
\hline RADIO-RX-THRESHOLD & $-81 \mathrm{dBm}$ \\
\hline Radio rx SNR Threshold & $10.0 \mathrm{dBm}$ \\
\hline Routing protocol & AODV \\
\hline MAC protocol & IEEE 802.11 \\
\hline
\end{tabular}

\subsubsection{The overall energy consumption}

We compare the overall energy consumption of routing protocols with RNG-1, RNG-2 and Non-RNG as shown Figure 6. When the number of nodes increases, the overall energy consumption increases. The traffic load is the same as the previous simulation. Our simulation results show that before the number of nodes is 80 , the density of nodes in a system is small. So the comparison of the collision and energy consumption is not quite obvious before node is 80 . After 80 nodes, the performance of RNG-1 and RNG-2 is significantly better than that of Non-RNG. We observe that the performance of the overall energy consumption and the collisions is similar. Lower collisions can reduce energy consumption. RNG-1 has 
better performance than RNG-2 and RNG-2's performance is better than Non-RNG's.

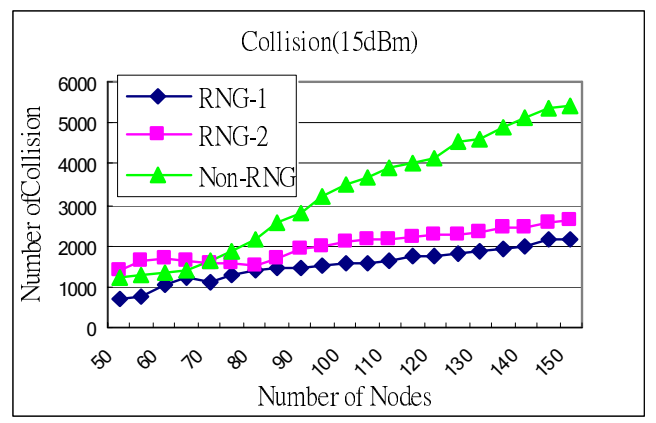

Figure 5. Collision comparison of RNG-1, RNG-2 and Non-RNG for AODV.

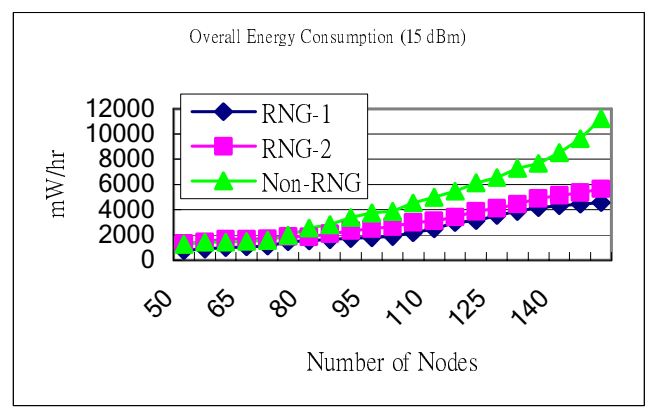

Figure 6. The overall energy consumption comparison of RNG-1, RNG-2 and Non-RNG for AODV.

\section{Conclusion}

In this paper, we propose a mechanism based on Relative Neighborhood Graphs (RNG) to adjust the node degree in ad hoc networks. Using the transmission range determined by RNG may result in asymmetric links. The asymmetric link problem can be solved by two approaches. One is that each node only accepts messages from one node which is its RNG neighbor. The other is that whenever a node receives a broadcast message from its neighbor, it checks its information to ensure that it includes this neighbor. Our simulation results show that a larger node degree implies larger transmission collisions. However, a lower interference can reduce energy consumption. Our method can guarantee that each node has a path to any other node in the network and achieve the goal of minimizing the node degree, lower collisions, and saving power. The routing protocols of our mechanism outperform the original protocols.

\section{References}

[1]IETF RFC2501 - Mobile Ad hoc Networking (MANET): Routing Protocol Performance Issues and Evaluation Considerations.

[2]G. Toussaint, "The Relative Neighborhood Graph of Finite Planar Set", Pattern Recognition, vol. 12, no. 4, pp. 261-268, 1980.

[3] K. J. Supowit, "The Relative Neighborhood Graph, with an Application to Minimum Spanning Trees", Journal of the ACM (JACM), v.30 n.3, p.428-448, July 1983.

[4] X. Chen, M. Faloutsos and S.V. Krishnamurthy, "Power Adaptive Broadcasting with Local Information in Ad Hoc Networks", IEEE ICNP 2003.

[5]C. Tang, C. S. Raghavendra and V. Prasanna, "Energy EfficientAdaptation of Multicast Protocols in Power Controlled Wireless Ad HocNetworks," ISPAN 2002

[6]D.B. Johnson and D.A.Maltz, Dynamic source routing in ad hoc wireless networks, in: Mobile Computing, eds. T. Imielinski and H. Korth (Kluwer Academic, 1996) chapter 5, pp. 153-181.

[7]Charles E. Perkins, Ad Hoc Networking, Addison-wesley, 2001

[8]S. A. Borbash, and E. H. Jennings, "Distributed Topology Control Algorithm for Multihop Wireless Networks," In Proc. of IEEE Int. Joint Conference on Neural Networks, page 355-360, 2002.

[9]Esther Jennings and Clayton M. Okino, "Topology for efficient information dissemination in ad hoc networking," August 2001, submitted.

[10]T.S. Rappaport, Wireless Communications, Principles and Practices, Prentice Hall, 1996.

[11] GloMoSim, http://pcl.cs.ucla.edu/projects/glomosim/

[12]C. Perkins and E. M. Royer, "Ad-hoc on-demand distance vector routing", Mobile Computing Systems and Applications (WMCSA), 1999. Proc. of 2nd IEEE Workshop on, Feb. 1999, New Orleans, LA., Pages:90-100

[13]J.P. Linnartz, Narrow Band Land-Mobile Radio Networks, Artech House, 1993.

[14] J. E. Wieselthier, G. D. Nguyen, and A. Ephremides, "On the construction of energy-efficient broadcast and multicast trees in wireless networks," Proc. nineteenth Annual Joint Conference of the IEEE Computer and Communication Societies (INFOCOM), 2000.

[15] J. Wu and F. Dai, "A distributed formation of a virtual backbone in MANETs using adjustable transmission ranges", Distributed Computing Systems, 2004. Proceedings. 24th International Conference on, 24-26 March 2004, Pages:372 - 379

[16] Benjie Chen, Kyle Jamieson, Hari Balakrishnan, and Robert Morris, "Span: An Energy-Efficient Coordination Algorithm for Topology Maintenance in Ad Hoc Wireless Networks." In Proceedings of ACM MobiCom 2001, page 85-96, Rome, Italy, July 16-21 2001.

[17] L. Feeney and M. Nilsson, Investigating the energy consumption of a wireless network interface in an ad hoc networking environment, in Proceedings of IEEE INFOCOM, Anchorage, AK 2001.

* This work is partially supported by the National Science Council, Taiwan under the grant number NSC-93-2213-E-002-123. 